\title{
MATERIALES PARA EL ESTUDIO DE LOS PARLAMENTOS (II)*
}

POR

\author{
ROSARIO HERRERO GUTIERREZ
}

\begin{abstract}
SUMARIO
I. EsPaña: 1. Publicaciones parlamentarias: 1.1. Cortes antiguas: 862 a 1800. 1.2. Período constitucional: 1810 a 1982. 2. Reglamentos. 3. Biografías; otros: materiales. 4. Historia. Anexo--II. Alemania (R. F.): 1. Publicaciones parlamentarias. 2. Reglamentos. 3. Otros materiales. 4. Bibliografía.-III. GRAN BretaÑA: 1. Publicaciones parlamentarias. 2. Reglamentos. 3. Otros materiales. 4. Bibliografía. 5. Historia--IV. Italia: 1. Publicaciones parlamentarias. 2. Reglamentos. 3. Otros materiales. 4. Historia. 5. Bibliografía.-V. FrANCIA: 1. Publicaciones parlamentarias. 2. Reglamentos. 3. Otros materiales. 4. Historia. 5. Bibliografía.-VI. ObRAS dE REFERENCIA. Bibliografías.-VII. Tratados geNerales. Estudios comparativos.-VIII. Organizactones INTERNACIONALES PARLAMENTARIAS.
\end{abstract}

\section{A.LEMANIA (R. F.)}

\section{PUBLICACIONES PARLAMENTARIAS}

La documentación parlamentaria oficial de la República Federal Alemana, Bundestag y Bundesrat, está publicada por la Verlag Dr. Hans Heger, Bonn. Recoge toda la documentación desde 1949 en las siguientes colecciones:

\section{Bundestag}

1. Verbandlungen des Deutsches Bundestages

Comprende las actas de las sesiones del Parlamento y otros documentos parla-mentarios.

a) Stenographiscbe Bericbte.

1. Wahlperiode 1949-1953: Band 1-17 = 1.-282. Sitzung.

2. Wahlperiode 1953-1957: Band 18-38 = 1.-227. Sitzung.

* La parte I del presente trabajo se publicó en el número 18-19 de esta Revista. 
3. Wahlperiode 1957-1961: Band 39-49 = 1.-168. Sitzung.

4. Wahlperiode 1961-1965: Band 50-59 = 1.-198. Sitzung.

5. Wahlperiode 1965-1969: Band 60-70 =1.-247. Sitzung.

6. Wahlperiode 1969-1972: Band 71-80 = 1.-199. Sitzung.

7. Wahlperiode 1972-1976: Band 81-99 $=1 .-259$. Sitzung.

8. Wahlperiode 1976-1980: Band 100 y ss. = 1.-ss. Sitzung.

Contiene el desarrollo in extenso de las sesiones plenarias del Bundestag, de acuerdos adoptados, de preguntas, etc.

Están numeradas correlativamente correspondiendo a cada número los períodos legislativos respectivos. Se encuadernan cuando alcanzan el tamaño de un volumen. Cada volumen tiene índice.

b) Drucksachen (Anlagen zu den Stenograpbiscben Bericbten).

1. Wahlperiode 1949-1953: And-Bd. 1-25 = Drs. Nr. 1-4682.

2. Wahlperiode 1953-1957: And-Bd. 26-54 = Drs. Nr. 1-3783.

3. Wahlperiode 1957-1961: And-Bd. 55-75 = Drs. Nr. 1-3007.

4. Wahlperiode 1961-1965: And-Bd. 76-100 = Drs. Nr. IV/1-3799.

5. Wahlperiode 1965-1969: And-Bd. 101-133 = Drs. Nr. V/1-4695.

6. Wahlperiode 1969-1972: And-Bd. 134-167 = Drs. Nr. VI/1-3831.

7. Wahlperiode 1972-1976: And-Bd. 168-227 = Drs. Nr. VII/1-5953.

8. Wahlperiode 1976 y ss.: And-Bd. 228 y ss. = Drs. Nr. VIII/1 y ss.

Todas las leyes, mociones, interpelaciones, preguntas verbales, peticiones, informes de las comisiones se imprimen y distribuyen como materia parlamentaria impresa. Están numeradas consecutivamente por cada período de legislatura. Al principio del cuarto período legislativo, el número del documento está precedido por el número del período legislativo en curso (por ejemplo: IV $/ \ldots, \mathrm{V} / \ldots$ ). Las materias impresas se encuadernan después de alcanzar el tamaño de un volumen. Se le añade un índice.

\section{c) Register zu den Verbandlugen des Deutschen Bundestags = Sacbregister und Sprecbregister.}

Indice de actas de sesiones del Bundestag; índice de materias (Sachregister) y de intervenciones de los miembros de la Cámara (Sprecbregister).

1. Wahlperiode 1949-1953.

2. Wahlperiode 1953-1957.

3. Wahlperiode 1957-1961.

4. Wahlperiode 1961-1965.

5. Wahlperiode 1965-1969.

6. Wahlperiode 1969-1972.

7. Wahlperiode 1972-1976.

Del primero al séptimo período legislativo hay un volumen de índice de materias $\mathrm{y}$ un volumen de «Index Speaker» para cada período.

Para el octavo período hay un segundo volumen de índice de materias y un segundo volumen de «Index Speaker».

Los documentos oficiales de las sesiones y los materiales impresos se pueden obtener encuadernados después de la publicación de cada número o encuadernar después de reunir un volumen.

Tiene la distribución exclusiva de estas publicaciones Verlag Dr. Hans Heger, de Bonn. Existe también en microficha. 
Los índices de las actas y otros documentos se publican anualmente; los índices de materias son por orden alfabético, y el índice de Speaker se hace también por orden alfabético: los nombres de todos los miembros que han hablado en las sesiones plenarias. Bajo los nombres está ordenada por materias de cada intervención, siguiendo el número de sesión y páginas.

\section{Amtliches Handbucb des Deutschen Bundestages... Wablperiode}

Publicación complementaria, útil para el trabajo parlamentario por su contenido como veremos. Dirigido por Deutschen Bundestag, Rheinbreitbach, Neue Darmstädter Verlagsanstalt.

1. Wahlperiode 1949-1953: no se ha publicado ningún manual oficial. (Para este período se puede consultar Die Volksvertretung. Handbucb des Deutscben Bundestages, publicado por Fritz Sänger, Stuttgart, Cotta, 1949, o Handbuch des Deutschen Bundestages, publicado por Fritz Sänger, Stuttgart, Cotta, 1952, y también Abschliessender Nacbtrag. 1, Wablperiode, en Handbuch des Deutscben, publicado por Fritz Sänger.)

2. Wahlperiode 1953-1957: véanse también cinco suplementos 1954-1957.

3. Wahlperiode 1957-1961: véanse también cuatro suplementos 1958-1961.

4. Wahlperiode 1961-1965: véanse también cuatro suplementos 1962-1965.

5. Wahlperiode 1965-1969: véanse también tres suplementos 1967-1969.

6. Wahlperiode 1969-1972: véanse también cinco suplementos 1970-1972.

7. Wahlperiode 1972-1976: véanse también cinco suplementos 1973-1976.

8. Wahlperiode 1976-1980: véase también un suplemento 1977.

Al comienzo del tercer período legislativo se publica en una edición de hojas sueltas. Solamente de la segunda a la quinta legislatura se publica una edición encuadernada. Los suplementos para ambas ediciones se han publicado y se siguen publicando: para la edición de hojas sueltas, generalmente con dos suplementos anuales; para la edición encuadernada, con un suplemento anual.

El Handbuch contiene: leyes, normas de procedimiento; resultados de las elecciones precedentes, organización y administración del Bundestag; lista de los miembros del Gobierno Federal; notas biográficas del Bundestag.

\section{BUNDESRAT}

1. Verhandlungen des Bundesrates

(Actas de sesiones del Bundesrat)

a) Stenograpbische Berichte (Relación de actas de sesiones).

1949-1950 = 1-43 sesión.

$1951=44-75$ sesión.

$1952=76-98$ sesión.

$1953=99-117$ sesión.

$1954=118-134$ sesión.

$1955=135-151$ sesión.

$1956=152-169$ sesión.

$1957=170-186$ sesión.

$1958=187-200$ sesión.

$1959=201-213$ sesión. 


$$
\begin{aligned}
& 1960=214-227 \text { sesión. } \\
& 1961=228-239 \text { sesión. } \\
& 1962=240-252 \text { sesión. } \\
& 1963=253-264 \text { sesión. } \\
& 1964=265-277 \text { sesión. } \\
& 1965=278-290 \text { sesión. } \\
& 1966=291-303 \text { sesión. } \\
& 1967=304-318 \text { sesión. } \\
& 1968=319-333 \text { sesión. } \\
& 1969=334-346 \text { sesión. } \\
& 1970=347-360 \text { sesión. } \\
& 1971=361-375 \text { sesión. } \\
& 1972=376-388 \text { sesión. } \\
& 1973=389-400 \text { sesión. } \\
& 1974=401-415 \text { sesión. } \\
& 1975=416-429 \text { sesión. } \\
& 1976=430-442 \text { sesión. } \\
& 1977=443-453 \text { sesión. }
\end{aligned}
$$

La publicación de estas actas es palabra por palabra, no resumida. Están numeradas correlativamente desde la primera sesión, en 1949, hasta la actualidad. Al final de cada año se encuadernan las actas, con índices anuales.

b) Drucksachen (Documentos).

$$
\begin{aligned}
& 1949=\mathrm{n} \cdot{ }^{\circ} 1 / 49-451 / 49 \text {. } \\
& 1950=\text { n. }{ }^{\circ} 1 / 50-1095 / 50 \text {. } \\
& 1951=\mathrm{n}^{\circ} 1 / 51-815 / 51 \text {. } \\
& 1952=n .01 / 52-506 / 52 \text {. } \\
& 1953=\mathrm{n} \cdot 0^{\circ} 1 / 53-537 / 53 \text {. } \\
& 1954={ }^{\circ}{ }^{\circ} 1 / 54-455 / 54 \text {. } \\
& 1955=\mathrm{n} .^{\circ} 1 / 55-438 / 55 \text {. } \\
& 1956=\mathrm{n} .^{\circ} 1 / 56-510 / 56 \text {. } \\
& 1957=\mathrm{n} .^{\circ} 1 / 57-496 / 57 \text {. } \\
& 1958=\mathrm{n} .{ }^{\circ} 1 / 58-320 / 58 \text {. } \\
& 1959=\mathrm{n} \cdot{ }^{\circ} 1 / 59-437 / 59 \text {. } \\
& 1960=n^{\circ} 1 / 60-432 / 60 \text {. } \\
& 1961=\mathrm{n} \cdot{ }^{\circ} 1 / 61-433 / 61 \text {. } \\
& 1962=\mathrm{n} \cdot{ }^{\circ} 1 / 62-435 / 62 \text {. } \\
& 1963=n^{\circ} 1 / 63-574 / 63 \text {. } \\
& 1964=n^{0} 1 / 64-562 / 64 \text {. } \\
& 1965=\mathrm{n} .01 / 65-606 / 65 \text {. } \\
& 1966=\mathrm{n} .01 / 66-542 / 66 \text {. } \\
& 1967=n .^{\circ} 1 / 67-651 / 67 \text {. } \\
& 1968=\mathrm{n} .{ }^{\circ} 1 / 68-719 / 68 \text {. } \\
& 1969=n .^{\circ} 1 / 69-687 / 69 \text {. } \\
& 1970=\text { n. } 1 / 70-741 / 70 \text {. } \\
& 1971=\mathbf{n} .{ }^{\circ} 1 / 71-744 / 71 \text {. } \\
& 1972=\mathrm{n} \cdot{ }^{\circ} 1 / 72-661 / 72 \text {. } \\
& 1973=\mathrm{n} .01 / 73-700 / 73 \text {. } \\
& 1974=\mathrm{n} .{ }^{\circ} 1 / 74-852 / 74 . \\
& 1975=\text { n. }{ }^{\circ} 1 / 75-791 / 75 \text {. } \\
& 1976=\mathrm{n} .^{\circ} 1 / 76-749 / 76 \text {. } \\
& 1977=\mathrm{n} \cdot{ }^{\circ} 1 / 77-662 / 77 \text {. }
\end{aligned}
$$


La documentación parlamentaria del Bundesrat se imprime parcialmente (en el caso de las leyes del Gobierno) o íntegramente (decretos). Están numeradas consecutivamente dentro de cada año (ejemplo: 272/72). Desde 1953 se publica una relación de materias impresas conteniendo números y títulos de éstas.

c) Sach- und Sprechregister

(Indices de materias y de Speaker).

1949-1952 (un volumen).

1953-1976 (un volumen por año).

Estos índices se publican anualmente. El índice de materias está ordenado de manera sistematizada y alfabética; cubre el total de actas y documentos parlamentarios de las discusiones del Bundesrat durante el año en curso. El índice de Speaker, por orden alfabético, los nombres de quienes han intervenido en sesiones plenarias del Bundesrat.

2. Handbuch des Bundesrates (cuarta edición, dirigido por el Bundesrat, Rheinbreitbach, Neue Darmstädter Verlagsanstalt, 1977)

Se actualiza continuamente con suplementos. Contiene legislación, organización y administración del Bundesrat, biografías, etc.

\section{REgLAMENTOS}

Los reglamentos del Bundestag y sus sucesivas modificaciones están recogidos en la publicación anteriormente mencionada: Amtlicbes Handbuch des Deutschen Bundestags..., 1949 a 1979. Igualmente, los reglamentos del Bundesrat están incluidos en el Handbuch des Bundesrates.

Independientemente de las publicaciones singulares de los reglamentos, aparece recogido en el Boletín de Legislación Extranjera, publicado por las Cortes (España), desde 1910, y en la publicación oficial legislativa Bundesgesetzblatt.

Finalmente, en algunas ediciones colectivas, como I rigolamenti parlamentari (Milán, Giuffrè, 1953), aparece el Reglamento del Bundestag, aprobado el 6 de diciembre de 1951, pp. 167-200, y el Reglamento del Bundesrat, aprobado el 8 de septiembre de 1950 , pp. 207-214, o en algunas de las publicaciones que detallaremos a continuación bajo el epígrafe de «otros materiales».

Son numerosos los estudios que se han realizado sobre los Reglamentos del Parlamento de Alemania Federal; estudios que giran generalmente sobre la constitucionalidad de los mismos, constitucionalidad que está sometida al Tribunal Constitucional.

Aunque nos estamos refiriendo en todo este capítulo a Alemania Federal, daremos alguna referencia a algún comentario de Reglamentos de los Reichtags sólo a título comparativo:

- ARndt, K. F.: Parlamentarische Geschäftsordnungsautonomie und autonomes parlamentsrecbt, Berlín, 1966.

- Bernau: Die Verfassungsrecbtliche Bedeutung von Geschäftsordnungen oberster Staatsorgane, Gotinga, 1954.

- Cicconetrr, Stefano Maria: Regolamenti parlamentari e giudizio de Costituzionalita nel diritto italiano e comparato (Stati Uniti, Germanie Federale, Italie), Padua, Cedam, 1979.

Estudia, por una parte, los reglamentos parlamentarios dentro del ordena- 
miento jurídico de Alemania Federal y el control jurisdiccional de legitimidad constitucional.

- CraushaAR: Die Bebandlung von Reichsratseinsprüchen im Reichstag und Gescbäftsordnungshagen. Ein Streitfall aus der Staatsrecbtlicben Praxis, en «Archiv des Öffentlichen Rechts», v. 10 (1926).

- HaAgen: Die Rechtsnatur der Parlamentariscben Geschäftsordnung mit besondere Berücksicbtigung der Gescbäftsordnungen des Preussiscben Landtags und des Reichtags, Breslau, 1929.

- Mangoldt-Flein: Das Bonner Grundgesetz, Berlín, etc., 1966.

- MонL: Kritiscbe Erörterungen über Ordnung und Gewobnbeiten des deutscben Reiches, II: Die Verbandlungen im Reichtage, en «Zeitschrift für die gesamte Staatswissenschaft» (1875).

- MüNCH: Grundgesetz-Kommentar, Munich, 1976.

- Perels: Das autonome Reichstagsrecht, Berlín, 1903.

- ReIfEnBerg: Die Bundesverfassungsorgane und ibre Gescbäftsordnungen, Gotinga, 1958.

- RITZEL-BüCKER: Handbuch für die parlamentarische Praxis, Francfort del Main, 1970.

- RITZEL-Zoch: Gescbäftsordnungen des deutscben Bundestages bescblossen am 6 Dezember, 1951, Francfort del Main, 1952.

- Schack: Die Prüfung der rechtsmässigkeit von Gesetz und Verordnung, Berlín, 1918.

- SchneIDER: Die Bedeutung der Gescbäftordnungen oberster Staatsorgane für das Verfassunsleben, en Festschrift für Rudolf Smend, Gotinga, 1952.

- SCHWEITzer: Aktuelle probleme des parlamentarischen Gescbäftordnungrechts, en «Neue juristische Wochenschrift» (1956).

- Vogler: Die Ordnungsgewalt der deutschen Parlamente, Hamburgo, 1926.

\section{OTROS MATERIALES}

Son numerosas y bastante completas las publicaciones de algunos manuales que aportan numerosos datos sobre organización, funcionamiento, proceso legislativo, producción legislativa, etc., del Parlamento alemán. Unas están publicadas por el propio Parlamento: Deutscher Bundestag Presse- und Informationszentrum, y otros por editores particulares. Destacamos de entre ellas:

- Alemanta (R. F.). Bundestag: 30. Jabre Deutscher Bundestag. Dokumentation Statistik Daten, Bearbeitet von Peter Schindler, Bonn, 1979, 504 p.

Contiene todos los acontecimientos, datos, cifras, estadísticas, etc., del Bundestag desde 1949 a 1979 . Elecciones (leyes, fecha de celebración, descripción de los partidos, resultados, etc.). Miembros del Parlamento (edad, clase social, permanencia en otras legislaturas, formación escolar y universitaria). Presidencia, dirección y ältestenrat (elección, trabajo, etc.). Grupos parlamentarios (estadísticas, variaciones, trabajos, etc.). Parlamento y Gobierno (coaliciones, oposición, ministros, etc.). Sesiones plenarias (estadísticas, trabajos). Comisiones (presidentes, estadísticas, comisiones de investigación, de encuesta, etc.). Legislación y procedimiento de control (estadísticas). Modificación de la ley fundamental (documentación). Legislación sobre Derecho parlamentario. Modificaciones de Reglamento. Casos de aplicación del Reglamento. Inmunidades. Peticiones. Reuniones del Bundes. Relaciones internacionales y europeas (Unión Interparlamentaria, Parlamento Europeo, Conferencia de Presidentes 
Parlamentarios Europeos, OTAN, etc.). Presupuestos. Administración (publicaciones de los servicios científicos). Prensa y trabajos de asuntos públicos.. Asociaciones parlamentarias.

Contiene, además, índice de las fuentes e índice de materias.

\section{Publicaciones del Bundestag}

I. Los servicios científicos del Bundestag editan las siguientes publicaciones:

a) Materialien, serie publicada desde 1965 hasta 1979.59 títulos, entre ellos:

1. Verwaltungskontrolle durch Parlamentsbeauftragte im Ausland Ombudsman, 1965,83 p. (Este número está actualizado en el cuaderno núm. 17, 1970.)

2. Wissenschaftlicbe Dienste der Verwaltung des Deutschen Bundestages, 1966,. 20 p. (Actualizado en el cuaderno núm. 54.)

3. Die Organisationsformen der Auslandskulturarbeit ausgewählte Staaten,. 1966, 82 p.

4. Parlamentariscbe Fachausdrücke Deutsch-Französisch, 1967, 22 p.

Los restantes títulos pueden consultarse en Bundestag, 30. Jabre... ellos:

b) Bibliograpbien, desde 1962 a 1978.59 bibliografías de varios temas, entre

1. Bibliograpbie zum Notstandsrecht, 1962, 89 p. (actualizada en los núms. 5* 12 y 28).

2. Bibliograpbie zum Bund-Länder-Verbältnis, 1963, 43 p. (actualizada en el núm. 13).

3. Bibliograpbie zur Stellung und zum Recht Parlamentarischer Untersuchungsausschüsse, 1963, $22 \mathrm{p}$.

4. Bibliograpbie zur Situation der Frau in Beruf, Familie und Gesellschaft, $1963,33 \mathrm{p}$.

La relación completa de las bibliografías aparece publicada en Bundestag, 30. Jabre...

c) Veröffentlichte Gesetzesmatialien.

1. Verwaltungsverfabrensgesetz (VwVfG) vom 25 mai 1976 (BGBL. I. S. 1253).

2. Gesetz über den Vollzug der Freibeitsstrafe und freibeitsentziebenden Massregeln der Besserung und Sicherung-Strafvollzugsgesetz (StVollozG) vom 16 März 1976 (BGBL. I. S. 581) (Dez. 1976).

La relación completa, en Bundestag, 30. Jabre...

d) Fundstellennacbweise zu den Gesetzesmaterialien der Bundesgesetze.

1. Sozialgesetzbuch (SGB)-Allgemeiner Teil vom 11. Dezember 1975 (BGBL. I. S. 3015).

2. Sozialgerichtsgesetz (SGG) vom 3. September 1953 (BGBL. I.S. 1239) (Nov. 1976).

- La relación completa en Bundestag, 30. Jabre... 
c) Sacb- und Sprecbregister.

Estos índices de los debates y otros documentos del Bundestag comprenden por períodos:

Indice de materias (un volumen): 1 a 7 Wahlperiode, 1949-1976.

Indice de autores (un volumen): 1 a 7 Wahlperiode, 1949-1976.

Indice de materias (un volumen): a partir del 8 Wahlperiode, 1976.

Indice de autores (dos volúmenes): a partir del 8 Wahlperiode, 1976.

Indices de debates y otros documentos del Bundesrat:

Indice de autores y materias (un volumen): 1 Wahlperiode, 1949-1952.

Indice de autores y materias por año (un volumen): 2 a 7 Wahlperiode, 1953-1976.

f) Stand der Gesetzgebung des Bundes (GESTA).

Esta publicación comprende colección de hojas intercambiables y tomos completos:

Hojas intercambiables: 7 Wahlperiode, 1972-1976.

Tomos completos: 7 Wahlperiode, 1972-1976.

Hojas intercambiables: 8 Wahlperiode, 1976-1980.

g) Informationsbandbucb des Bundes (IHB).

Comprende datos sobre el Gobierno, Bundestag y Bundesrat. Se inicia en 1978; es de hojas intercambiables.

h) Findbücber zu den Aktenbeständen des Parlamentsarcbivs.

Comprende:

1. Inventario 1: Der Zonenberirat der Britisch Besetzten Zone (1946-1949). 1973.

2. Inventario 10: Der Vermittlungsausschluss des Deutschen Bundestag und des Bundesrates (1950-1961). 1977.

3. Inventario 2: Der Wirtscbaftsrat des Vereinigten Wirtscbaftsgebietes (19471949). 1979.

i) Hauptabteilung Wissenschaftlicbe Dienste.

II. Al departamento Presse- und Informationszentrum del Bundestag le compete toda información sobre los trabajos del Parlamento y comisiones. También se le encomienda el cuidado de las visitas del Bundestag y Reichstagsgebäudes en Berlín. Para todo ello realiza publicaciones en serie de servicios de información, trabajos en serie, monografías, etc. Por ejemplo, Wolfang Kessel, Bundesversammlug Wie unser Staatsoberbaupt Gewäblt Wird, 1979.

III. Las asociaciones parlamentarias del Bundestag suelen publicar sus actividades, publicaciones científicas, etc.:

a) Deutsche Parlamentarische Gesellschaft e. v.

b) Kommission für Geschichte des Parlamentarismus und der politischen Parteien e. $v$. ha publicado:

1. Beiträge zur Geschicbte des parlamentarismus und der politiscben Parteien (hasta 1979, 65 tomos). 
2. Quellen zur Gescbicbte des Parlamentarismus und der politiscben Parteien (hasta 1979, 16 teils en varios tomos).

3. Bibliographien zur Gescbicbte des Parlamentarismus und der politiscben Parteien (hasta 1979, 8 volúmenes).

4. Handbuch der Gescbichte des Deutschen Parlamentarismus (hasta 1979, un volumen).

c) Interparlamentarische Arbeitsgemeinschaft (IPA). Sus publicaciones son sobre derecho y organización del Parlamento.

d) Verband der Parlaments- und Verhandlungsstenographen e. v. Publica Neue Stenographische Praxis (trimestral).

e) Deutsche Vereinigung für Parlamentsfragen e. v. Además de publicar seminarios, coloquios, sobre cuestiones actuales del Parlamento, publica Zeitschrift für Parlamentsfragen (trimestral).

f) Vereinigung ehemaliger Mitglieder des Deutschen Bundestages e. v.

\section{Handbücber des Deutscben Bundestages:}

a) Edición oficial:

Amtliches Handbucb des Deutsches Bundestages, hrsg. vom Deutschen Bundestagsverwaltung. Neue Darmstädter Verlagsanstalt. (E1 contenido aparece descrito en el capítulo de publicaciones parlamentarias.)

b) Ediciones no oficiales:

Handbuch des Deutschen Bundestags, hrsg. con Fritz Sänger, Stuttgart (Cotta, 1957: Klett). Comprende:

1. Wahlperiode, 1949 (con el subtítulo «Die Volksvertretung»), 2. ${ }^{\text {a }}$ ed., 1952.

2. Wahlperiode, 1953.

3. Wahlperiode, 1957.

Kürscbners Volkshandbucb Deutscher Bundestag, Darmstadt (Neue Darstädter Verlangsanstalt). Comprende:

2. Wahlperiode, 1953-57.

3. Wahlperiode, 1957.

4. Wahlperiode, 1961.

5. Wahlperiode, 1965.

6. Wahlperiode, 1969.

7. Wahlperiode, 1972.

8. Wahlperiode, 1976.

V. Veröffentlichungen des statistischen Bundesamtes. Se han publicado las siguientes:

1949 Die Bundestagswabl am 14 August 1949. Statistik der Bundesrepublik Deutschland, Stuttgart-Colonia, 1952 (10 vols.).

1953 Die Wabl zum 2. Deutschen Bundestag, am 6 September 1953, StuttgartColonia, 1954-1955 (2 vols.).

1957 Die Wabl zum 3. Deutscben Bundestag am 15 September 1957, Stuttgart, 1957-1959 (4 vols.).

1961 Wabl zum 4. Deutschen Bundestag am 17 September 1957, StuttgartMaguncia, 1962-1964 (4 vols.). 
1965 Wabl zum 5. Deutscben Bundestag am 19 September 1965, en Fachserie A, Bevölkerung und Kurtu, Reihe 8, Stuttgart-Maguncia, 1964-1967 ( 9 vols.).

1969 Wabl zum 6. Deutschen Bundestag am 28 September 1969, en Fachserie A, Bevölkerung und Kultur, Reihe 8, Stuttgart-Maguncia, 1969-1971 ( 8 vols.).

1972 Wabl zum 7. Deutscben Bundestag am 19 November 1972, en Fachserie A, Bevölkerung und Kultur, Reihe 8, Stuttgart-Maguncia, 1972-1975 (9 vols.).

1976 Wabl zum 8. Deutschen Bundestag am 3 Oktober 1976, en Fachserie A, Bevölkerung und Kultur, Reihe 8, Stuttgart-Maguncia, 1977-1978 (9 vols.).

1978 Wabl zum 9. Deutscben Bundestag.

\section{Otros materiales}

Alemania (R.F.): Hessischer Landta. 1-8 Wablperiode, 1949, Darmstadt, Neue Darmstädter Verlagsanstalt, 1952 y ss. (en la colección Volkshandbuch). Contiene datos sobre biografías de los parlamentarios, presidente, grupos parlamentarios, «Ailtestenrat», comisiones, trabajos de Landtags, Reglamento de Hessischen Landtags (y proporciona la referencia en la publicación oficial, por ejemplo, el reglamento de 1 de diciembre de 1978, en GVBL, I. S. 694), formación de las leyes, ley sobre la «Rechtsverhältnisse» de los diputados del Hessischen Landtags (ejemplo: GVBL. Hessisches Abgeordnetengesetz-Hess $\mathrm{AbgG}$ ), ley sobre las elecciones sobre Landtag des Landes Hessen (ejemplo: 1974, Landtagswahlgesetz-LWG, 10 de enero de 1974, en GVBL, I. S. 41), se completa con una estadística de las elecciones por distritos y partidos.

- BuEcker, Joseph: Handbuch für die parlamentarische Praxis, Francfort del Main, Kommentator, 1981. Contiene la legislación y reglamentos siguientes: Reglamento de la Dieta Federal alemana, Ley fundamental de la República Federal, Ley de control de actas parlamentarias, Ley sobre la situación jurídica de los miembros de la Dieta Federal, Ley de la comisión de protección, Ley sobre el comisionado parlamentario para el ejército, preceptos de Derecho civil y penal que hacen referencia a miembros de la Dieta y que pueden ser significativos para los parlamentarios, extracto de la Ley sobre el Tribunal Constitucional Federal, Reglamento del Consejo Federal (Bundesrat), Reglamento del Gobierno Federal, Reglamento de la comisión conjunta del artículo 53.a de la Ley fundamental, Reglamento conjunto de la Dieta Federal y el Consejo Federal para la comisión de mediación prevista en el artículo 77 de la Ley fundamental, estatuto del grupo de trabajo interparlamentario, comentario al Reglamento de la Dieta Federal (en apéndices incluye Reglamento de trabajo del grupo parlamentario CDU/CSU, del SPD, del FDP).

- Roemring, Hans-Helmut: Handbuch des Deutschen Parlamentarismus. Das Regierungssystem des Bundesrepublik in 270 Stichworten, Munich, R. Piper, 1970. Es un manual que, aunque científico, va destinado a un público amplio para dar a conocer el sistema parlamentario y gobierno de Alemania; en voces ordenadas alfabéticamente, recoge aspectos, evolución y problemas del Parlamento alemán, instituciones, Ley fundamental de Bonn. En anexo contiene fuentes y datos estadísticos: cuadro estadístico de la actividad del BT y BR 1949-1969, organización administrativa del Bundestag, comisiones permanentes del Bundestag, gobierno 1949-1969, Reglamento del Bundestag alemán con suplementos, reglamentos de los grupos del Bundestag: CDU/CSU, SPD, FDP. 
- Schmidt-Schmiedebach, H.: Datenbanken im Dienste der Gesetzgebung der Rechtsprechung und der Verwaltung, en «Almanack 1970», Colonia, 1970 y siguientes.

- ThaYSEN, L.: Parlamentarisches regierungssustem in der Bundesrepublik Deutscbland. Daten-Fakten-Unteile im Grundiss, 2 Aufl, Opladen, Leske und Budeich, 1976.

\section{BIBLIOGRAFIA *}

Achterberg, Norbert: Die Parlamentarische Verbandlung, Berlín, Duncker \& Humblot, 1979.

- Grundzüge des Parlamentsrechts, Munich, 1971.

AHRENS, Wolf-Eberhard: Inmunität von Abgeordneten, Bad Hamburg, Althenaeum, 1970.

Baumgartner, Serge: Die Standesinitiative. Eine Untersuchung der recbtlichen und politischen Funktion dieses föderalistischen Mitwirkungsrechts, Basilea, Helbing \& Lichtenhahn, 1980.

BoDENHEIM, Dieter G.: Kolision parlamentarischen Kontrollrechte. Zum verfassungsrecbtlichen Verbältnis von parlamentariscben Frage- und Untersuchungsrecht, Hamburgo, Joachim Heirmann, 1979.

BoeHert, Carl: Test und Prüfung von Gesetzentwürfen, Colonia, Carl Heimanns, 1980.

Borgs-MacIEJEwSKI, Hermann: Parlamentsorganisation, Institutionen des Bundestages und ibre Aufgaben, Heidelberg, Deckers's, 1980.

CaRsten: Princes and Parliaments in germany from the 15th the 18th Century, 1959.

CASSESE, A.: Parliamentary control over foreign policy legal Essays, Alphen aan den Rijn, Sijthoff \& Noordhoff, 1980.

Deverlein (Comp.): Der Reichstag: Aufsätze, Protokolle und Darstellungen zur Geschichte der Parlamentarischen Vertretung des deutscben Volkes, 1871-1933, Francfort, 1963.

FrIEDRICH, Manfred: Landesparlamente in der Bundesrepublik, Opladen, Westdeutscher, 1975.

Friesenhahn, Ernst: Parlement und Regierung im modernen Staat, Berlín, Walter de Gruyter, 1958.

KISSLER, Leo: Die Öffentlicbkeitsfunktion des Deutscben Bundestages. Theorie Empirie-Refam, Berlín, Duncker \& Humblot, 1976.

KluXeN, Kurt (ed.): Parlamentarismus, Colonia, etc., 1969 (amplia bibliografía).

Kremer, Klemens: Parlamentsauffösung. Praxis, Tbeorie Ausblick, Colonia, Carl Haymanns, 1974.

LIPPHARDT, Hanns-Rudolf: Die Kontingentierte Debatte. Parlamentsrechtliche untersucbung zur Redeordnung des Bundestages..., Berlín, Duncker und Humblot, 1976.

Loewenberg, Gerhard: Parlamentarismus im politiscben system der Bundesrepublik Deutscbland, Tubinga, 1969.

LutTERBeck, Bernd: Parlament und Information. Eine informationstheoretische und verfassungsrecbtlicbe Untersuchung, Munich, etc., R. Oldenbourg, 1977 (contiene

* Incluye algunas obras de carácter general que proporcionan a su vez bibliografía. Se ha procurado recoger, además, estudios sobre aspectos particulares de Derecho parlamentario alemán, historia sobre el Bundestag, Bundesrat, etc., que facilitan un conocimiento global sobre el Parlamento alemán, así como nuevas referencias bibliográficas, por considerar que puede resultar útil esta selección bibliográfica al margen de las exhaustivas bibliografías reseñadas en el capítulo correspondiente. 
discusiones actuales de las relaciones del Gobierno-Parlamento; aplicación de métodos, enfoques informáticos, etc.; incluye una amplia bibliografía).

Magiera, Siegfried: Parlament und Staatsleitung in der Verfassungsordnung des Grundgesetzes. Eine Untersucbung su den Grundlagen der Stellung und Aufgaben des Deutscben Bundestages..., Berlín, Duncker und Humblot, 1979.

MorSCHER, Siegbert: Die Parlamentarische Interpellation, Berlín, Duncker \& Humblot, 1973.

Odewald, Jens: Der Parlamentarische Hilgsdienst in den Vereinigten Staten von Amerika und in der Bundesrepublik Deutscbland, Berlín, Duncken und Humblot, 1976 (contiene la descripción de los servicios de apoyo a los parlamentarios: documentación, asesores jurídicos, etc.).

Parlamentsaufloesung. Praxis-Tbeorie-Ausblick. Herausgegeben von Klemens Kremer. Mit Beiträgen von Gerald Kretschmer (y otros), Colonia, etc., Carl Heymanns, 1974.

Rausch, Heinz: Bundestag und Bundesregierung. Eine Institutionenkunde, Munich, Verlag C. H. Beck, 1976.

RITTER (Comp.): Gesellschaft Parlament und Regierung: Zur Gescbicbte des Parlamentarismus und der Politischen Parteiens in Deutschland, Düsseldorf, 1974.

STEIGER, Heinhard: Organisatoriscbe Grundlagen des parlamentarischen Regierungssystems. Eine Untersuchung zur rechtlichen Stellung des Deutschen Bundestages..., Berlín, Duncker und Humblot, 1973 (monografía sobre fuentes jurídicas de Derecho parlamentario y constitucional).

Stengel, Karl: Die Parlamentsdienste im Bundestag. Entstebung. Arbeitsweise und Verfassungsrechtliche Grundlage, Berna, etc., Paul Haupt, 1977.

Thaysen, Uwe: Parlamentsreform in Theorie und Prasis zur institutionellen lemfäbigkeit des Parlamentarischen Regierungs systems. Eine empirische Analyse Parlamentsreform im 5. Deutschen Bundestag, Opladen, Westdeutscher Verlag, 1972 (obra esencialmente didáctica, renuncia a una exposición de método y notas para presentar los datos, hechos y valoraciones de la práctica parlamentaria como un conjunto de materiales de enseñanza).

Trossmann, Hans: Parlamentsrecht des Deutschen Bundestages, Munich, C. H. Beck, 1977.

Tsatsos, Dimitris: Die Unzulässigkeit der Kumulation von Bundestags- und Bundesratmandat, Tubinga, J. C. B. Mohr (Paul Siebeck), 1965.

VonDERBECK, Hans-Josef: Parlamentarische Informations- und Redebefugnisse, Berlín, Duncker und Humblot, 1981.

WIDDER, Helmut: Parlamentarische Strukturen im politischen System, Berlín, Duncker und Humblot, 1979.

Sobre la organización del Bundestag y del Bundestat, pueden consultarse:

Bundesrat als Verfassungsorgan und politische Kraft, en Festschrift zum 25. Jäbrigen Bestehen des Bundesrates, Darmstadt, Bad Honnel, 1974.

DeverLeIn, Ernst: Föderalismus. Die Historiscben und Pbilosopbiscben Grundlagen des föderativen Prinzips, Bonn, 1972 (Schriftenreihe der Bundeszentrale für politische Bildung, Heft 94).

KlatT, H. (ed.): lit. Der Bundestag im Verfassungsgefüge der Bundesrepublik Deutschland, Bonn, Presse- und Informationszentrum des Deutschen Bundestages, 1980.

LAUfER, Heinz: Der Bundesrat, Bonn, 1972.

- Das föderative System der Bundesrepublik Deutscbland, 3 Af., Munich, 1977.

Lohmar, Ulrich: Das Hobe Haus, Der Bundestag und die Verfassungswirklicbkeit, Stuttgart, 1975. 
NeunReITHer, Karlheinz: Der Bundesrat zwischen Politik und Verwaltung, Heidelberg, 1959.

PFiTZER, Albert: Der Bundesrat, 29 Auf., Bonn, 1979.

RapP, Alfred: Der Bundesrat. Blick Aut 25. Jahre Bundesrat, Stuttgatt, 1974.

SCHAEFER, Friedrich: Der Bundestag. Eine darstellung seiner Aufgaben und seiner arbeitsweise, 3 Verbesserte und erweiterte Auf., Opladen, Westdeutscher Verlag, 1977.

SCHAEFER, Hans: Der Bundesrat, Colonia, 1955.

SCHÜLE, Adolf: Die Informationsflicht der Bundesregierung gegenüber dem Bundesrat, en Carl Bilfinger zum 75. Geburtstag, Colonia, 1954.

SüsterHENN, Adolf: Senats- oder Bundesratssystem?, en Staats- und Verwaltungswissenschaft Beiträge, Speyer, 1957.

Tsatsos, Dimitris: Die Unzulässigkeit der Kumulation von Bundestag- und Bundesratsmandat, Tubinga, 1965.

Vitsthum, Wolfgang Graf: Parlament und Plannung. Zur Verfassungsgerechten Zuordnung der Funktionen von Bundesregierung und Bundestag bei der politischen Planung, Baden-Baden, Nomos, 1978.

VonDERBECK, Hans-Josef: Der Bundesrat ein Teil des Parlaments der Bundesrepublik Deutscbland?, Meisenheim, 1964.

ZeH, Wolfgang: Der Deutsche Bundestag, 3 Auf., Düsseldorf, Droste Verlag, 1979 (contiene información sobre el régimen parlamentario en Alemania [R. F.]; Derecho electoral; miembros, órganos, grupos parlamentarios; sesiones plenarias, comisiones; participación del Bundestag en las instituciones exteriores al Parlamento; órganos de cooperación; comisión de mediación).

Ziller, Gebhard: Der Bundesrat, 6 Auf., Düsseldorf, Droste, 1982 (contiene datos sobre historia del Bundesrat; función: colaboración del Bundesrat en la legislación y administración de los Länder; reuniones, pleno, presidente, comisiones; procedimiento en el Bundesrat; relaciones con el Bundestag, etc.).

\section{GRAN BRETAÑA}

\section{PUBLICACIONES PARLAMENTARIAS}

Las publicaciones parlamentarias inglesas son publicadas por Her Majestaty's Stationery Office (HMSO) y por Hansard; su uso no es fácil, por lo que existen guías que facilitan su utilización, entre ellas John E. Pemberton, British Official Publications, y S. G. Ollé, An Introduction to British Government Publications.

HMSO divide sus publicaciones en dos grupos: publicaciones parlamentarias y no parlamentarias. A veces resulta difícil determinar en cuál de las dos categorías se encuentra enmarcada una publicación.

Las publicaciones parlamentarias comprenden actas de los debates y documentos:

A) Parliamentary Papers * (publicados por HMSO) son aquellos documentos producidos o requeridos por el Parlamento en el curso de su trabajo: «Bills», «Records of the Proceeding in the House», «Information Papers», etc., tanto de la House of Commons como de House of Lords. Cada documento parlamentario tiene su propio número individual, por lo que estos documentos se ordenan por este número en grupos según el contenido de los mismos. Estos grupos son: House of Commons

* Para el siglo xvi, «Journals of the House of Commons» (desde 1547), «Journals of the House of Lords» (desde 1509); hasta 1800, «House of Commons Papers» (1731-1800), «House of Lords Papers» (1788-1801). 
Papers, House of Commons Bills, House of Lords Papers and Bills y Command Papers.

B) Parliamentary Debats (publicados por Hansard) tecoge los debates de las sesiones del Parlamento.

Para localizar las publicaciones más recientes de Papers y Debats se acude a los índices de las publicaciones del Gobierno: diarios, semanales, mensuales, anuales, cada cinco años, etc., publicados por HMSO.

Se publica una lista diaria, Daily List, de las publicaciones del Gobierno, que incluye tanto las publicaciones parlamentarias («House of Iords and Commons Papers and Bills», «Command Papers», «Acts and Debats») y las publicaciones no parlamentarias.

El Daily List se reemplazó por un catálogo mensual titulado Government Publications. Contiene en la primera parte: a) listas de «House of Lords and House of Commons Papers and Bills», ordenadas por el número de orden; b) lista de «Command Papers», por orden numérico; c) lista de «Acts» y «Debats» publicadas durante el mes. En la segunda parte: lista de publicaciones parlamentarias y no parlamentarias (excluyendo el contenido de la primera parte); esta lista está dividida por departamentos gubernamentales, incluyendo todas las noticias publicadas por cada uno de ellos durante ese mes con detalles de sus precios y el «ccimmand numbers» o «lords or commons papers numbers». Contiene un índice por materias, títulos, autores y chairmen.

A su vez, esta publicación mensual es sustituida por un catálogo anual, llamado Government Publications, desde 1900. Cada cinco años se publica un índice (19711975, 1976-1980, etc.). La paginación de estos catálogos es continua para el período de los cinco años.

El contenido de los catálogos anuales y de cinco años el siguiente:

- Listas de publicaciones parlamentarias, ordenadas por sesiones, y éstas por orden numérico por «Bills», «Papers» y «Command Paper».

- Lista clasificada de publicaciones parlamentarias y no parlamentarias (excluyendo el apartado anterior). Esta lista está ordenacla por el nombre del departamento del Gobierno que produce la publicación.

- Lista de publicaciones periódicas publicadas por la HMSO, con precios incluidos.

- Indice alfabético, con entradas de títulos, materias, autores y presidentes de las comisiones.

Por último, diremos que existen índices de períodos más amplios:

- Períodos de diez años: General Alpbabetical Index to the Bills, Reports and Papers Printed by Order of the House of Commons and the Reports and Papers Presented by Command (General Index 1900-1949).

- Indices de la documentación parlamentaria del siglo XIX.

Otros indices de documentos

Puede verse:

- Gran Bretaña. Stationary Office, Government Publications. Catalogue, 1923.

- The Sales Catalogues of Britisb Government Publications, 1836-1921, Dobbs Fony (N. Y.), Oceana Publications, 1974, 4 vols.

- Sessional Index of House of Commons Bills, Papers and of Commons Papers. Para cada sesión y cada cinco años desde 1800. Desde la sesión de 1979-1980 se ha mecanizado este Sessional Index en la base de datos POLIS (Parliamentary On-Line Information System). 
Cada biblioteca o centro de documentación que recibe y posee la colección completa de las publicaciones parlamentarias procura recogerla en volúmenes conteniendo todo el material producido durante un período legislativo. Estos volúmenes son conocidos como Sessional Papers o Sessional Sets.

En el caso de la House of Commons, esta disposición convencional de los documentos se divide en cuatro grandes grupos:

- Bills. Comprende «House of Commons Bills» (borradores de las «Public Bills», dispuestos por orden alfabético de los títulos de los «Bills»).

- Reports from Committees. Comprende «House of Commons Papers» (informes de las comisiones o «House of Commons Committees»).

- Reports from Commissioners. Comprende «House of Commons Papers and Command Papers» (informes de otras comisiones, «Royal Commissions», etc.).

- Accounts and Papers. Comprende «House of Commons Papers and Command Papers» (informes estimados como «White Papers», series de tratados y otros papeles de Estado).

Independientemente de como está ordenada en una biblioteca o centro de documentación la documentación parlamentaria, ya sea por Cámaras, y dentro de ellas por categorías, comprendiendo períodos legislativos (Sessional Sets), o bien a la inversa, por categorías abarcando ambas Cámaras, vamos a detallar a continuación el contenido de esta documentación, su forma de citarla y localización dentro de las publicaciones de HMSO y de Hansard.

\section{Command Papers}

Esta documentación es la usada con más frecuencia. Contiene informes importantes del Gobierno, algunos informes de la Law Commission y los informes de la Royal Commission:

- «Statements of Government Policy» (o «White Papers»).

- «Statistical» o informes anuales (algunos son considerados como publicaciones no parlamentarias).

- «Reports of Royal Commissions».

- «Reports of Committees» (algunos pueden no ser publicados como publicaciones no parlamentarias).

- «Reports of Tribunals of Enquiry».

- «State Papers» (incluye la serie de tratados).

A los Command Papers se les da a cada uno un número individual precedido por una abreviatura de la palabra «Command». Esta abreviatura y el número se imprimen en el ángulo izquierdo de la cubierta del informe. Estos números continúan de un período legislativo a otro.

Antes de 1956 se usaban distintas abreviaturas de la palabra «Command», entre ellas:

- 1st series 1833-1869 [1]-[4222] (en esta primera serie no aparece la abreviatura de «Command»).

- 2nd series 1870-1899 [C. 1]-[C. 9550].

- 3rd series 1900-1918 [Cd. 1]-[Cd. 9239].

- 4th series 1919-1956 [Cmd. 1]-Cmd. 9889.

- 5th series 1956-date Comd. 1 . 
Como se puede observar, a partir de 1922 se suprimen. los corchetes.

Si las publicaciones están ordenadas por sesiones o petíodos, o están encuadernadas dentro de las Sessional Sets, será necesario conoces: la fecha del «Command Papers»; para ello se debe consultar The Concordance of Command Papers 18331972, impresos en Pemberton; hecha esta consulta, se averigua en qué volumen de la Sessional Sets está y a continuación se consulta el Sessional Index, y allí se encontrará el número del C.ommand, indicando volumen y página dentro de la Sessional Sets, donde puede ser encontrado y consultado.

$\mathrm{Si}$, por el contrario, se conoce el número y la abreviatura, se determina la fecha de publicación, y se acude después a los índices anuales de Government Publications.

Los State Papers se encuadernan juntos al final del Sessional Sets (en volúmenes titulados Accounts and Papers). Dentro de los State Papers se encuentran los Treaty Series: son Command Papers; cada uno tiene un número de Command, pero a cada uno se le añade su número propio de la serie de tratados:

- Si no están encuadernados dentro de Sessional Sets, se puede colocar toda la serie de los Treaty juntos. Hay índices por series anuales y cada tres o cuatro años.

- También aparecen en los catálogos de HMSO de Government Publications, en donde se dan el número de serie y el número de Command Paper.

HMSO publicó en 1970 un Index of British Treaties 1101-1968, compilado por Clive Parry y Charity Hopkins. En el volumen 1 tiene entrada por materias, y en los volúmenes 2 y 3 , por fecha de lo tratado.

\section{Bills}

Los Bills o proyectos de ley son presentados ante el Pärlamento para su discusión y aprobación; antes de llegar a ser Acts, pasa por una serie de etapas, cuyos resultados se van imprimiendo con tipología característica para cáda uno de ellos, por lo que a continuación pasamos a detallarlas:

$1{ }^{a}$ First reading (Primera lectura). Es una primera lectura puramente formal del título de la ley por el «Clerk of the House». Despuśs de esta lectura, la ley se imprime por HMSO y se pone a la venta.

2. ${ }^{a}$ Second reading (Segunda lectura). Se discute el contenido de la «Bill». El debate es publicado por Hansard en Weekly Hansard.

3. a Committee Stage (Etapa de comisiones). La Cámara en pleno puede formar una comisión para examinar el «Bill». Generalmente, los detalles de los «Bills» se someten al «Standing Committee» para su discusión. Por ello Hansard publica separadamente Parliamentary Debates, House of Commons, Official Reports of Standing.

Committees Debates se publica diariamente. Los impiesos diarios se reemplazan por volúmenes encuadernados. Cuando se quiere localizar en qué comisión se discutió un determinado «Bill», se consulta el Government Publications Catalogue, bajo el encabezamiento «House of Commons: Minutes of Proceeding» (hasta 1975, y desde 1976, «Standing Committee...»).

4. ${ }^{\mathrm{a}}$ Report Stage (Dictamen). Cuando un «Bill» ha sido enmendado por la «Standing Committee» se da a la Cámara una oportunidald para considerar los cambios, pudiendo ser devuelto de nuevo a la comisión.

5. ${ }^{\text {a }}$ Third reading (Tercera lectura). Con una discusión menos detallada pasa a la «House of Lords» para su aprobación.

6. ${ }^{a} \quad$ Lords Stage. El «Bill» se reimprime cuando pasa a la «House of Lords» para su aprobación. A cada «Bill» se le da un número, que aparece en el ángulo izquierdo de la primera página entre corchetes, si es un «House of "Commons Bill»; si aparece entre paréntesis indica un «House of Lords Bill». 
Las iniciales H. C. o H. L. se usan para indicar las dos diferentes Cámaras del. Parlamento y se da también la sesión parlamentaria. Por tanto, una cita del «Bill». dará:

- Iniciales de la «Houes».

- La sesión del Parlamento.

- El número de «Bill» entre corchetes [Commons] o entre paréntesis (Lords).

Ejemplo: H.L. 1976-77 (172).

Si hay pocas enmiendas a un «Bill», se publica sólo la enmienda, llevando el mis-. mo número de «Bill» original, pero añadiendo una letra minúscula. Ejemplo: $(50 \mathrm{~b})$.

Si se ha enmendado mucho el texto, se reimprime el texto y se le da un nuevo número.

Si ha tenido varias enmiendas, se publica una lista de éstas, que tiene el mismo. número del «Bill» original, pero añadiendo un número romano. Ejemplo: (148 III).

La mayor parte de «Bills» requiere la aprobación de ambas Cámaras del Parlamento; en este caso, se reimprime de nuevo con otro número cuando ha pasado a la otra Cámara para ser aprobado.

¿Dónde se pueden localizar los «Bills» más recientes? Tanto los «Bills» como las. enmiendas, en el Daily List Government Publications.

Los debates que tienen lugar en la «Committee Stage» pueden ser encontrados. en Official Report of Standing Committees.

Los debates en pleno de la Cámara son recogidos por Hansard.

Cuando un «Bill» recibe el «Royal Assent», ésta es reseñado en publicaciones tales como New Law Journal, Current Law, etc.

Una vez recibido el «Royal Assent», una copia de la «Act» se imprime y aparece en el Daily List.

Cuando se trata de viejos «Bills», si se tiene encuadernados las «Sessional Sets» y en ellas todos los textos de todas las sesiones de los «Bills», junto con sus enmiendas, se encontrará por orden alfabético en los volúmenes titulados «Bills» al comienzo de la «Sessional Set».

Si no están encuadernados, todas las versiones del «Bill» publicadas pueden consultarse al comienzo de los catálogos anuales del Government Publications.

\section{Papers of House of Lords and House of Commons}

Los House of Lords Papers and Bills se publican en secuencia numérica común, tanto de «Papers» como de «Bills» mezclados. El número de cada documento se im-. prime entre paréntesis al pie de la cubierta principal. La cita es: H. L. Sesión/ número.

Los House of Commons Papers se imprimen con secuencia numérica de los «Commons Bills». La cita incluye: H. C. Sesión/número.

Si están encuadernados dentro de las «Sessional Sets», en el índice se localizan. el volumen y la página.

\section{Parliamentary Debates *}

En 1803 se publican los primeros «reports» semioficiales por William Cobbert. El siguiente impreso fue de Hansard.

* Para los siglos xvir y xvin se publican dos series: «A Collection of Parliamentary Debates» (17th and 18th Century), «Sessional Acts Printed in Black Letter» (1702-1868). 
Se han publicado cinco series de Parliamentary Debates:

$1 .^{\mathrm{a}}$ 1803-20.

$2 .^{\mathrm{a}} \quad 1820-30$.

$3 .^{a} \quad 1830-91$.

4. $.^{\mathrm{a}} 1892-1908$.

$5 a^{a} 1909$.

Desde 1909, la «House of Commons» publica Officidl Reports of Parliamentary Debates y «House of Lord» publica separadamente sus debates.

Los Official Report of Parliamentary Debates se publican: a) diariamente (Daily Parts), y estas publicaciones diarias son reemplazadas por volúmenes conteniendo los períodos o sesiones parlamentarios, con índice de la sesión (Bound Volume of Debates); b) se publica también un Weekly Hansard (publicación semanal), conteniendo todos los «reports» diarios en una única publicáción, y un Weekly Index para los debates.

En las citas para los debates se da generalmente: número de volúmenes/Cámara/ serie/fecha/número de columna. Las referencias en los índices son a los números de la columna, no a la página.

Si el número está impreso en cursiva, es una referencia a las preguntas escritas que están en una secuencia separada al final del volumen y no a la columna de los -debates.

Los índices tienen entradas por materias y por nombres de los Speakers. Estos indices están en el último volumen encuadernado de los Parliamentary Debates.

Las discusiones sobre un «Bill» tienen entrada bajo el nombre del «Bill». Las abreviaturas $1 \mathrm{R}, 2 \mathrm{R}, 3 \mathrm{R}$ indican la primera, segunda y tercera lecturas.

Sobre el contenido de las publicaciones parlamentarias se han editado algunas guías, como la de D. Marshallsary y J. H. Smith, Ford List of British Parliamentary Papers, 1965-74, Nendelen, Liechtenstein, KTO Press "(Southampton University Studies in Parliamentary Papers).

\section{Otras guías}

Bond, Maurice F.: Guide to the Record of Parliament, Londres, HMSO, 1971.

Brooke, John: General Index to Reports from Committees of the House of Commons, 1715-1801, Bishops Short Ford, Chadwyckhealy, 1973.

Cornelius, I. V.: Britisb Government Publications: An Introductory Guide, Stirling University Library, 1973.

ForD, G.: Select list of Reports and Others Papers in the Journal of the House of Commons, 1688-1800, Nendeln, KTO Press, 1976.

Ford, G., y Ford, Percy: Breviate of Parliamentary Papers, 1900-1916, Oxford, Blackwell, 1957; 1917-1939, Oxford, 1951; 1940-1954, Oxford, 1961.

- A Guide to Parliamentary Papers: What the Are, How to find them, How to use them, 3. a ed., Shannon, Irish University Press, 1972.

- Select list of British Parliamentary Papers, 1833-1899, Oxford, Blackwell, 1953.

Ford, G.; Ford, Percy, y Marshallsary, Diana: Select list of British Parliamentary Papers, 1955-1964, Irish University Press, 1970.

FORD, G., y FORD, Percy: List of British Parliamentary Papers, 1965-1974 together with Specialist Commentaries, Nendeln, KTO Press, 1979.

Hansard's Catalog and Breviate of Parliamentary Papers, 1696-1834, Oxford, Blackwell, 1953.

HugHes, Christopher J.: The British Statute Book, Londres, Hutchinson's University Library, 1957.

Moys, Elizabeth M. (ed.): Manual of Law Librarianship: The use and Organization of legal literature, Boulder (Col.), Westview Press, 1976. 
Rodgers, Frank, y Phelps, Rose B.: A Guide to Parliamentary Papers, Urbana, University of Illinois Graduate School of Library Science, 1967.

RoDGERs, Frank: Serial publications in the British Parliamentary Papers, 1900-1968: A bibliograpby, Chicago, American Library Association, 1971.

\section{REGLAMENTOS}

Para el estudio del complejo procedimiento parlamentario británico es necesario conocer su contenido y sus fuentes.

Sobre su contenido solamente indicaremos que puede ser dividido en tres grupos:

- «Forms» de procedimiento usadas en cada Cámara. (Ejemplo: procedimiento sobre «Bills» en sus varias etapas.)

- Organización de dirección y delegación establecido para cada una de ellas. (Ejemplo: reglas sobre presidencia y «Permanent Officers» de cada Cámara.)

- Reglas que regulan el trabajo de las «Forms» y organización.

Las reglas del procedimiento parlamentario tienen rasgos que pertenecen a diferentes períodos y etapas históricas; de ahí que el procedimiento moderno esté formado por un conjunto de estratos históricos que se combinan con reglas y costumbres modernas.

Como es sabido, una parte del procedimiento está codificada y otra no: procedimiento escrito y procedimiento no escrito o consuetudinario.

La parte escrita, codificada, es simplemente un complemento a la no escrita, son los Standing Orders (Reglamento permanente), que son dictados por la autoridad de la Cámara y publicadas, para la Cámara de los Comunes con las actualizaciones de las enmiendas, en dos volúmenes: 1) relativo a «Public Business», y 2) relativo a «Private Business», publicados por HMSO.

Para la Cámara de los Lores, la parte consuetudinaria forma una gran parte del conjunto del procedimiento. Los «Standing Orders» de la Cámara de los Lores también se publican en dos volúmenes: 1) relativo a los «Public Business», y 2) relativo a «Private Bills». (Mientras que los «Standings Orders» de ambas Cámaras forman, más o menos, un código, en la Cámara de los Comunes las S.O. de «Public Business» son simplemente modificaciones de las reglas de procedimiento consuetudinario; a partir de 1907, los «Standing Orders» de la Cámara de los Comunes se han elaborado de modo circunstancial, aunque una gran parte de las reglas de debate siguen estando reguladas exclusivamente por la costumbre.)

La parte de procedimiento no escrita, «Practice», en terminología legal es el «equivalente al procedimiento». Muchos datan de los siglos XVII y xvIII, por ejemplo, las relativas a los privilegios de los «Peers» y «Lords». Algunas, muy pocas, se modifican por los S. O. para acelerar el proceso.

Una relación de la práctica consuetudinaria en la Cámara de los Lores está recogida en The Companion to the Standing Orders, compilada por los «Officers» de la Cámara y distribuido con la autorización de la «Procedure Committee».

Para la Cámara de los Comunes, un resumen del procedimiento llamado The Manual of Procedure in the Public Business, recopilado para uso de los miembros y revisado periódicamente.

Las fuentes sobre las que están basadas las reglas de procedimiento son, por orden de importancia: 1) la costumbre; 2) los «Standing Orders», «Ocasional Orders» y resoluciones de cada Cámara; 3) «Rulings» del «Chair», y 4) «Statute» (más raramente). 
La primera parte: «Practice», práctica consuetudinaria, es aquella parte del procedimiento que se desarrolla espontáneamente en el transcurso del tratamiento de los asuntos en cada Cámara. Hay viejas prácticas que se conocen como «Ancient Usages»; no necesitan más prueba de autenticidad que su existencia de facto.

Hay otras prácticas autorizadas deliberadamente: se dictan dentro de la Cámara en forma de «Resolutions» $\mathrm{u}$ «Orders» y están recogidas en los «Journals» (en la Cámara de los Comunes desde 1547; en la Cámara de los Lores desde 1509). La «Practice» fue desarrollada por precedentes: cuando un caso no podía ser decidido con alguna norma existente, la Cámara indicaba a la comisión que buscase en los «Journals» los precedentes que sobre el caso pudieran existir en los que se hayan decidido casos semejantes.

Para el conocimiento de la vieja práctica es necesario el estudio de los citados «Journals» y los «Records» de debates publicados en las series de Hansard's Reports o bien en las fuentes manuscritas. Junto a la práctica antigua existe otra moderna que aplican los «Ancient Usages» y se armoniza con los «Standing Orders», formando el cuerpo general de procedimiento.

La segunda fuente de procedimiento son los «Standing Orders», que se componen de unas reglas con una dirección limitada o permanente. Desde el comienzo del período de «Standing Orders» se pueden encontrar «Orders» y «Resolutions» hechos por la Cámara de los Comunes con efecto permanente, por ejemplo, la «Order» de 1836 prohibiendo la impresión y publicación de lo debatido en una comisión antes de haber sido informado por la Cámara.

La tercera fuente son los «Ruling» de «Chair». Consiste en las decisiones dadas por el Speaker; entre otras, dar contestación a las preguntas sobre el aumento de puntos del orden. Todas las decisiones pueden ser localizadas en el «Official Report of Debates». Algunas veces se han dado decisiones privadamente, no habiendo oportunidad de ser deliberadas públicamente en la Cámara. Ambas decisiones, públicas o privadas, se recogen en el curso de cada sesión por el «Clerk» de la Cámara e impreso de vez en cuando para uso oficial.

Finalmente, la parte del procedimiento que se apoya en «Statute» tiene una menor importancia, a excepción de un primer «Statute» regulando puestos en los Lores, y otras como Act de 1850; Private Legislation Procedure (Scotland) Act 1936; Statutory Orders (Special Procedure) Acts 1945 y 1965; Statutory Instruments Act 1946, y The Parliament Acts 1911 y 1948. Exchequer and Audit Departments Acts 19681922; The Public Accounts and Charges Act 1891, y The Provisional Collection of Taxes Acts 1913 y 1968.

Para la localización de las reglas de procedimiento parlamentario británico, escrito y consuetudinario, además de las publicaciones y actas de sesiones anteriormente mencionadas, puede consultarse:

- Catalogue of Government Publications, de HMSO (mensual o anual).

- Catalogue of British Official Publications not Published by HMSO, Londres, Chadwyckhealey (anual).

- House of Commons Bulletin, Journals, etc.

- Statutory Instrument, Statutory Publications Office (véase catálogo HMSO).

La base de datos del Parlamento británico, Parliamentary On Line Information System (POLIS), proporciona referencia sobre «Proceeding» de ambas Cámaras.

-_ Boletin de Legislación Extranjera. España: Congreso de los Diputados (mensual).

- Informations Constitutionnelles et Parlementaires. Suiza: Unión Interparlamentaria (trimestral). 
Son muchos los estudios sobre el procedimiento parlamentario inglés. Sobre los siglos xvir y xviII, Smith, De republica Anglorum, Londres, 1560; Privileges and Practices of Parliament, Londres, 1628; Lambad, The Orders, Proceeding, Punisbments and Privileges of Parliament, Londres, 1792. Estudios posteriores sobre los orígenes de procedimiento en general: Hansard Society for Parliamentary Government, Lords and Commons: How Parliament Began and How it Works, Londres, The Hansard Society, 1954; S. L. Shakdher, Comparative Study of Financial Procedure in Parliament in U.K. and India, Nueva Delhi, Government of India Press, 1968; Alfred Bossom, Our House: An Introduction to Parliamentary Procedure, Londres, Barrie, 1948. Estudios para los años 1880-1882: Richard A. Chapman, The significance of Parliamentary Procedure, en «Parliamentary Affairs», primavera 1963, 179-187; Albert Henry Hanson y Herbert B. Wiseman, Parliament at Work: $A$ Case Book of Parliamentary Procedure, Londres, Stevens, 1962; Edward Hughes, The Changes in Parliamentary Procedure, 1880-1882, en Essays presented to Sir Lewis Namier, Londres, Macmillan, 1956, pp. 287-319. Para 1970-1971: David Millar, The Process of legislation: Second Report from the Procedure Committee, 1970-71, en «The Table», 40 (1971), 73-78; Edward T. Winterton, Orders of the Day, Londres, Cassell, 1953.

Sobre procedimiento en la Cámara de los Comunes: C. J. Boulton, Recent Developments in House of Commons Procedure, en «Parliamentary Affairs», 21 (1969), 61-71; Norman Henry Brasher, The House of Commons: Some Procedural Problems, en Studies in Britisb Government, $2 .^{\text {a }}$ ed., Londres, Macmillan, 1971, pp. 90-108; Gilbert Francis Campion, An Introduction to the Procedure of the House of Commons, 3. ${ }^{\mathrm{a}} \mathrm{ed}$, Londres, Macmillan, 1958; Edward Fellowes, Practice and Procedure in the House of Commons 1919-1961, en «Journal of the Parliaments of the Commonwealth», 43 (1961), 105-114; John Hatsell, Precedents of Proceeding in the House of Commons, en Shannon, 5 vols., Irish University Press, 1971-1972; Joseph Redlich, The Procedure of the House of Commons: A Study of its History and Present Form, Londres, A. Constable, 1908.

Para el estudio del procedimiento en la Cámara de los Lores: Henry Burrows, Standing Orders of the House of Lords Relative to Private Bills, etc., en «The Table», 17 (1948), 67-135.

Sobre los «Standing Orders»: Gerald John Wheeler, The Practice of Private Bills, with the Standing Orders of the House of Lords and House of Commons, and Rules as to Provisional Orders, Londres, Shaw and Sons, 1900; Orlo C. Williams, The Historical Development of Private Bill Procedure and Standing Orders in the House of Commons, 2 vols., Londres, HMSO, 1948-1949; F. W. Lascelles, Procedure and the Standing Orders, en «Parliamentary Affairs», 7 (1953-1954), 88-95.

Sobre los «Statutory Instruments»: J. T. Craig, The Working of the Statutory Instruments Act, 1946, en «Public Administration», 39 (1961), 181-191.

\section{OTROS MATERIALES}

Independientemente de las numerosas bibliografías sobre el Parlamento británico reseñadas en el capítulo general de obras de referencia y bibliografías, se cuenta con otros materiales que ayudan a completar el estudio del Parlamento británico.

Por un lado, instituciones: las propias Cámaras reúnen o publican materiales sobre el Parlamento. La Cámara de los Comunes ha reunido una colección en 92 volúmenes de folletos de interés histórico, material político del periodo de 1559-1740: Parliamentary Collection; también publica anualmente House of Commons Library Documents, y semanalmente, House of Commons Weekly Information. Bulletin 
(asuntos tratados y por tratar en la Cámara, partidos, etc.). Por su parte, la Cámara de los Lores publica trimestralmente Library Bulletin, de importancia por la riqueza de manuscritos que posee esta biblioteca.

La asociación parlamentaria Commonwealth Parliamentary Association (Londres), a través de su Parliamentary Information and Reference Centre, se encarga de reunir y conservar una colección de libros y materiales especializados sobre parlamentos de la Commonwealth y estudios comparativos sobre cien legislaturas (ya se han procesado en 1982 más de tres mil documentos). Este centro depende del apoyo directo de los Speakers, parlamentarios y bibliotecarios del Parlamento. El propósito es tener una única base de datos accesible a los parlamentarios, funcionarios parlamentarios y estudiantes interesados en el conocimiento de las instituciones parlamentarias; por otra parte, publica estudios monográficos.

Otra fuente de datos la proporcionan las revistas especializadas, que reseñan y publican listas de estudios sobre Parlamentos: Parliamentary Affairs, Parliamentarian, Legislative Studies Quarterly, Inter-Parliamentary Bulletin, Englisb Historical Review, Political Quarterly, The Table, Institute of Historical Research Bulletin, Political Studies, English Review, Modern Law Review, Public Law, Contemporary Review, History Today, Law Quarterly Review, Britisb Journal of Political Science, Political Science Quarterly.

Para la localización de tesis sobre trabajos del Parlamento británico, puede consultarse Roger R. Bilboul, Retrospective Index to theses of Great Britain and Ireland, 1716-1950 y Index to theses Accepted for Higher Degrees by the Universities of Great Britain and Ireland and the Council for National Academic Awards.

También puede consultarse Peter S. Bell, Dissertations on British History, 1815 1914: An Index to British and American Theses. Comprebensive Dissertation Index and Dissertation Abstracts International.

Algunas guías generales proporcionan datos de organización: Quintin M. Hailsham, Parliament, A Reader's Guide, Londres, Cambridge University Press, 1948; Christopher A. Leeds, Guide to British Government, 3.a ed., Swanage, Croxten Press, 1978; Francis M. G. Wilson, The Organization of British Central Government 1914-1964, 2. a ed., Londres, Allen and Unwin, 1968.

Datos estadísticos pueden verse en fuentes estadísticas como A. F. Conford y C. Loveless, Guide to Government Data: A Survey of Unpublished Social Science Material in Libraries of Government Departments in London, Londres, Macmillan, 1974; Directory of Research Resources in the United Kingdon for Business, Industry and Public Affairs, Londres, Library Associates, 1970; Richard Lawton (ed.), The Census and Social Structure: A Interpretative Guide to Nineteentb Century. Censuses for England and Wales, Londres, Frank Cass, 1978; W. F. Maunder (ed.), Reviews of United Kingdon Statistical Sources, 9 vols., Londres, Heinemann, 19741976; Brian R. Mitchell, Abstract of British Historical Statistics, Cambridge, Cambridge University Press, 1962; Stanley H. Palmer, Economic Arithmetic: A Guide to the Statistical Sources of English Commerce, Industry and Finance, 1700-1850, Nueva York, Genland Publishing, 1977; Kathleen G. Pickett, Sources of Official Date, Londres, Longmans, 1974; Graham Sergeant, A Statistical Sourcebook for Sociologist, Londres, Macmillan, 1972; Alan F. Sillitoe, Britain in Figures: A Handbook of Social Statistics, 2. ${ }^{\mathrm{a}}$ ed., Harmondsworth, Penguin, 1973.

Las biografías de los parlamentarios británicos pueden encontrarse en varios repertorios biográficos: Joseph O. Baylen y Norman J. Gossman (eds.), Biographical Dictionary of Modern British Radicals, Hassocks (Inglaterra), Harvester Press, 1979; The Business Background of MPS, Londres, Parliamentary Profiles, 1958; Dictionary of Labour Biograpby, Londres, Macmillan, 1972; Dictionary of National Biograpby, 73 vols., Londres, Oxford University Press, 1885-1960; Directory of the Corrent Governments of the United Kingdon and the Nations of the Commonwealth, Dallas, 
Political Research, 1979; Andrew Roth y Janice Kerbey, The MPs' Chart, Londres, Parliamentary Profiles, 1965; Peter Shipley, Directory of Pressure Groups and Representative Associations, 2. ${ }^{\text {a }}$ ed., Nueva York, Bowker, 1979; Michael Stenton, Who's Who of British Members of Parliament: A Biograpbical Dictionary of the House of Commons Based on Annual Volumes of Dod's Parliamentary Companion and otber Sources, Atlantic Highland (N. J.), Humanities Press, 1976; Dod's Parliamentary Companion.

Los diccionarios proporcionan datos, acontecimientos y varias clases de referencia: John A. Brendon (ed.), A Dictionary of British History, Londres, E. Arnold, 1937; Leonard W. Cowie, A Dictionary of British Social History, Londres, Bell, 1973; Louis Golding, Dictionary of Local Government in England and Wales, Londres, English Universities Press, 1962; Frank E. Huggett, A Dictionary of British. History 1815-1973; John Richardson, The Local Historian's Encyclopedia, New Barnet, Historical Publications, 1975; S. H. Steinberg y I. H. Evans (eds.), Stein berg's Dictionary of British History, 2. ${ }^{\text {a }}$ ed., Londres, E. Arnold, 1970.

Algunas fuentes sociales y económicas aportan datos de interés: Tessa Blackstone, Social Policy and Administration in Britain: A Bibliograpby, Londres, F. Pinter, 1975; W. J. Chaloner y R. C. Richardson, British Economic and Social History, Manchester, Manchester University Press, 1976; John H. Westergaard, Modern British Society: A Bibliograpby, 2. a ed., Londres, F. Pinter, 1977; Judith B. Williams, $A$ Guide to the Printed Material for English Social and Economic History, 1750-1850, 2 vols., Nueva York, Columbia University Press, 1926.

\section{BIBLIOGRAFIA}

Se trata aquí de dar una orientación bibliográfica de carácter general de los principales aspectos del Derecho parlamentario inglés y de cada una de ambas Cámaras.

Por lo que se refiere a estudios de carácter general, clasificados como libros de texto, la obra de William Ivor Jennings, Parliament, 2. ${ }^{\mathrm{a}}$ ed., Cambridge, Cambridge University Press, 1957, recoge diversos aspectos de organización y funcionamiento; sigue esta misma línea Kenneth R. Mackenzie, The English Parliament, Harmondsworth, Penguin Books, 1963.

Estudios clásicos como: Strathearn Gordon, Our Parliament, Londres, Cassell, 1964; Patrick Howarth, Questions in the House: The History of a Unique British Institution, Londres, Lane, 1956; Countrey P. Ilbert, Parliament: Its History. Constitution and Practice, $3 .^{a}$ ed., Londres, Oxford University Press, 1956; Roland A. Young, The British Parliament, III, Evanston, Northwestern University Press, 1962; Harold J. Laski, Reflections on the Constitution: The House of Commons, the Cabinet and the Civil Service, Nueva York, Viking Press, 1951.

Trabajos más modernos proporcionan una buena introducción al Parlamento inglés: Douglas E. Ashford, Policy and Politics in Britain: The Limits of Consensus, Oxford, Basil Blackwell, 1981; Kenneth Bradshaw y David Pring, Parliament and Congress, Newed-Londres, Quartet, 1981; William L. Miller, The end of British Politics?, Oxford, Clarendon Press, 1981; Timothy Raison, Power and Parliament, Oxford, Basil Blackwell, 1977; Michael Rush, Parliament and the Public, Londres, Longman, 1976; íd., Parliament Government in Britain, Londres, Pitman, 1981.

Sobre el proceso legislativo existen estudios muy completos, como los de John A. G. Griffith, Parliamentary scruting of Government Bills, Londres, Allen and Unwin, 1974; William Ivor Jennings, The Law and the Constitution, 5. ${ }^{a}$ ed., Londres, University of London Press, 1967; S. A. Walkland, The Legislative Process in Great Britain, Nueva York, Praeger, 1968. 
Sin duda, el trabajo más completo sigue siendo el de Thomas Erskine May, Treaties on the Law, Privileges, Proceedings and Usage of Parliament, 19 ed., editado por David Lidderdale, Londres, Butterwosth, 1976.

Para el estudio de la legislación delegada una buena monografía es la de John $\mathrm{E}$. Kersell, Parliamentary Supervision of Delegated Legislation: The United Kingdon, Australia, New Zealand and Canada, Londres, Stevens, 1960.

Sobre «Private Member Bills», es un buen trabajo el de Peter A. Bromhead, Private Member's Bills in the British Parliament, Londres, Routledge and Paul, 1956.

La bibliografía es abundante para el estudio de la organización del Parlamento:

Sobre la estructura de las comisiones, Alfred Morris (ed.), The Growth of Parliamentary Scrutiny by Committee: A Symposium, Oxford, Pergamon Press, 1970, o la obra de David L. Coombes The Scrutiny of Ministers Powers by the Select Committee on Nationalized Industries, en «Public Law», primavera 1965, 9-29.

Sobre el «Speaker», sus funciones, historia: Arthur Irwin Dasent, The Speaker of the House of Commons from the Earliest times to the Present Day... with a Portrait of Every Speaker Where one is Known to Exit, Londres, John Lane, 1911; Philip Laundy, The Office of Speaker, Londres, Cassell, 1964; Michael Macdonagh, The Speaker of the House, Londres, Methuen, 1914.

Sobre la reforma del Parlamento también existen numerosos estudios: John A. Cannon, Parliamentary Reform, 1640-1832, Cambridge, Cambridge University Press, 1973; Bernard R. Crick, The Reform of Parliament, 2. ${ }^{\mathrm{a}}$ ed. rev., Londres, Weidenfeld and Nicolson, 1970; id., Reform of the Commons, Londres, Fabian Society, 1959; Samuel Edward Finer, Adversary Politics and Electoral Reform, Londres, Wigram, 1975; Hansard Society for Parliamentary Government, Parliamentary Reform, 1933-1960: A Survey of Suggested Reforms, Londres, Cassell, 1961.

Sobre los miembros del Parlamento existen estudios de diversos aspectos sociales, económicos, etc.: Thomas H. S. Escott, Gentlemen of the House of Commons, 2 volúmenes, Londres, Hurst and Blackett, 1902; Colin Mellors, The British MP: A Socio-Economic Study of the House of Commons, Farnborough, Saxon House, 1978; Elizabeth Vallance, Women in the House. A Study of Women Members of Parliament, Londres, Athlone Press, 1979.

Finalmente, reseñamos los principales estudios sobre la Cámara de los Comunes y la Cámara de los Lores.

Para la Cámara de los Lores: Gilbert Francis Montriou Campion, Parliament: A Survey, Londres, Allen and Unwin, 1952; Philip Norton, The Commons in Perspective, Oxford, Martin Robertson, 1981; R. F. D. Palgrave, The House of Commons, Londres, Macmillan, 1869; Study of Parliament Group, The Commons in transition, Londres, Fontana, 1970; S. A. Walkland (ed.), The House of Commons in the Twentieth Century: Essays by Members of the Study of Parliament Group, Oxford, Clarendon Press, 1979.

Sobre las funciones de la Cámara de los Comunes puede verse la obra de Gilbert Francis Montriou Campion. 